Review began 10/14/2021 Review ended 10/14/2021 Published 10/19/2021 Exp. concern 04/07/2022

\section{Copyright 2021}

Alaithan et al. This is an open access article distributed under the terms of the Creative Commons Attribution License CC-BY 4.0., which permits unrestricted use, distribution, and reproduction in any medium, provided the original author and source are credited.

\title{
Pulmonary Embolism in COVID-19 Patients: A Retrospective Case-Control Study
}

Fatimah A. Alaithan ${ }^{1}$, Mahdi H. Aljawad ${ }^{2}$, Asia H. Ghawas ${ }^{3}$, Abdulrahman S. Althobaiti ${ }^{4}$, Qusai A. Almuslem ${ }^{5}$, Mohammed H. Bin Nasif ${ }^{6}$, Faisal F. Algharbi ${ }^{7}$, Reem A. Alshehri ${ }^{8}$, Yousef K. Al Gethami ${ }^{9}$, Khaled W. Altowayan ${ }^{6}$, Fahad K. Alzahrani ${ }^{6}$, Amal A. Suwaylih ${ }^{10}$, Abdullah S. Alwadai ${ }^{11}$, Abdulmajeed M. Badawi ${ }^{8}$, Malak Alshammari ${ }^{12}$

1. Radiology, Dammam Medical Complex, Dammam, SAU 2. Radiology, King Fahd University Hospital, Al-Khobar, SAU 3. General Practice, Dhurma General Hospital, Dhurma, SAU 4. General Practice, Al Faisaliah Primary Health Care Center, Dammam, SAU 5. General Practice, Al Naeriyah Hospital, Dammam, SAU 6. College of Medicine, King Saud University, Riyadh, SAU 7. College of Medicine, King Khalid University Hospital, Riyadh, SAU 8. College of Medicine, King Abdulaziz University, Jeddah, SAU 9. College of Medicine, Taif University, Al-Taif, SAU 10. College of Medicine, Hadhramout University, Al-Mukalla, YEM 11. College of Medicine, King Khalid University, Abha, SAU 12. College of Medicine, Imam Abdulrahman Bin Faisal University, Dammam, SAU

Corresponding author: Malak Alshammari, saudidoctor2020@gmail.com

\section{Expression of Concern}

Expression of Concern date: April 07, 2022. Cite this expression of concern as Alaithan F A, Aljawad M H, Ghawas A H, et al. (April 07, 2022) Expression of Concern: Pulmonary Embolism in COVID-19 Patients: A Retrospective Case-Control Study. Cureus 14(4): x9. doi:10.7759/cureus.x9.

The concern relates to the provenance of this article as brought to our attention by Faisal Alhawaj, who denies authorship of this article and others published in Cureus. These articles were submitted and subsequently published purportedly as an effort coordinated by Imam Abdulrahman Bin Faisal University to ensure all medical interns publish at least one peer-reviewed article in order to qualify for enrollment in a postgraduate residency program as stipulated by The Saudi Commission for Health Specialties (SCFHS).

The journal has not been presented with enough evidence to warrant the formal retraction of these articles as both Imam Abdulrahman Bin Faisal University and The Saudi Commission for Health Specialties have failed to respond to numerous communications requesting additional information regarding these allegations. While we acknowledge that the provenance of these articles is very much in question, we cannot act until these claims have been investigated by the appropriate institutions with the results of said investigation communicated to Cureus.

The concern and this note will remain appended to the above-mentioned article until Cureus is provided with official confirmation from Imam Abdulrahman Bin Faisal University or The Saudi Commission for Health Specialties.

\begin{abstract}
Background: Coagulopathy is a well-recognized complication in patients with coronavirus disease 2019 (COVID-19). Pulmonary embolism (PE) has substantial morbidity and mortality if the diagnosis is missed or the management is delayed. Computed tomography pulmonary angiography (CT-PA) is the imaging modality of choice for PE. Therefore, this study aimed to investigate the positive rate of CT-PA for PE among patients with COVID-19.
\end{abstract}

Methods: We conducted a retrospective study examining the diagnostic yield of CT-PA in patients with confirmed COVID-19 and compared it with that in patients without COVID-19. The study included all adult patients with confirmed COVID-19 who presented from June 2020 to June 2021.

Results: The study included 316 patients, including 158 patients with COVID-19, who underwent CT-PA for ruling out PE. Overall, 76 patients were found to have PE on the CT-PA scan, yielding a positive rate of $24.1 \%$, with a significant difference between patients with COVID-19 (8.2\%) and those without COVID-19 (39.9\%). Further, 138 (87.3\%) patients with COVID-19 had elevated D-dimer levels compared with 34 (21.5\%) patients without COVID-19. A multivariable regression analysis model revealed that the smoking status (odds ratio $[\mathrm{OR}]=1.94 ; 95 \%$ confidence interval $[\mathrm{CI}]: 1.4-3.8)$ and obesity $(\mathrm{OR}=4.1 ; 95 \% \mathrm{CI}: 1.5-8.9)$ were independent predictors of PE among patients with COVID-19. However, the elevated D-dimer level was not significantly associated with PE among patients with COVID-19 (OR = 0.7; 95\% CI: 0.4-1.8).

Conclusion: The study found that the positive rate of CT-PA for PE was lower among patients with PE indicating probable overutilization of investigation in these patients. Additionally, patients with COVID-19 had a higher proportion of elevated D-dimer levels that may be a contributor to the increased investigation for PE. Lastly, patients with COVID-19 who were current smokers had a higher tendency of having PE. 
Categories: Emergency Medicine, Internal Medicine, Radiology

Keywords: pulmonary angiography, computed tomography, pulmonary embolism, hyper coagulopathy, covid-19

\section{Introduction}

Coronavirus disease 2019 (COVID-19) is caused by severe acute respiratory syndrome coronavirus 2 (SARS$\mathrm{CoV}-2$ ) that was first identified as the pathogen responsible for a cluster of pneumonia cases in Wuhan, China, at the end of 2019. It was officially declared as a pandemic by the World Health Organization on March 11, 2020 [1]. The diagnosis of COVID-19 is made by nucleic acid amplification using reversetranscription polymerase chain reaction (RT-PCR) tests since its clinical features are non-specific. The most common presenting symptoms of COVID-19 include cough, fever, myalgia, headache, and dyspnea. While COVID-19 predominantly affects respiratory symptoms, the involvement of the organ systems is not uncommon [2]. Several studies highlighted the wide spectrum of complications seen in COVID-19, including cardiovascular, thromboembolic, neurologic, and inflammatory complications [3-5].

It became evident that patients with COVID-19 had a number of complex and varied coagulopathies predisposing them to a wide spectrum of thromboembolic conditions [6]. The pathogenesis of such coagulopathies is not well-understood. However, a number of changes in the prothrombotic coagulation factors have been reported in COVID-19, including elevated factor VIII, elevated fibrinogen, and increased viscosity [7]. Furthermore, there is evidence that the SARS-CoV-2 virus may invade the endothelial cells directly leading to cell injury and activating the coagulation cascade [8].

The spectrum of thromboembolic complications of COVID-19 is broad and ranges in severity from asymptomatic to organ dysfunction resulting in death [2]. Venous thromboembolism and pulmonary embolism (PE) are well-recognized complications of COVID-19, that were seen in up to $30 \%$ of patients in the intensive care unit according to initial reports [9]. Additionally, arterial thrombotic events such as stroke and myocardial infarction may occur, but they are less common than venous thrombosis in COVID-19 [10].

PE is a potentially life-threatening medical emergency. Early diagnosis and management defines the prognosis of $\mathrm{PE}$ as the mortality rate can reach up to $30 \%$ if the diagnosis of $\mathrm{PE}$ is missed or the management is delayed [11]. The clinical manifestations of PE are non-specific and have a significant overlap with those of COVID-19. Additionally, D-dimer levels are often elevated in patients with COVID-19, making it challenging to determine which patients require computed tomography pulmonary angiography (CT-PA) [12].

CT-PA is the diagnostic modality of choice for ruling out PE. Considering its widespread availability and high accuracy in making the diagnosis of PE, there is a low-threshold tendency for its overutilization. Hence, the positive rate of CT-PA for PE is often considered as an indicator of the appropriate use of the study. Limited studies have investigated the use of CT-PA in patients with COVID-19 in comparison with patients without COVID-19. Therefore, the aim of this study is to investigate the positive rate of CT-PA for PE among patients with COVID-19 in comparison to a control group.

\section{Materials And Methods}

\section{Study design and setting}

After obtaining approval from the ethics and research committee (IAU-IRB 2020-155), we conducted a retrospective study examining the diagnostic yield of CT-PA in patients with confirmed COVID-19 and compared it with patients without COVID-19. The study also aimed to identify the determinants of PE in patients with COVID-19. It was conducted at two major hospitals (Qatif Central Hospital and Dammam Central Hospital) in the Eastern Province of Saudi Arabia. In these institutions, the CT-PA scan can only be requested by board-certified physicians.

\section{Study population}

Eligible patients included all adult patients with COVID-19 confirmed by RT-PCR tests who underwent CT-PA for ruling out PE. Additionally, a similar number of patients without COVID-19 who underwent CT-PA were chosen randomly to be the control group. The hospital information system was utilized to identify all patients meeting the eligibility criteria who presented from June 2020 to June 2021 . These included a total of 316 patients, including 158 patients with COVID-19.

\section{Data collection and imaging technique}

A structured data collection form was used to collect the data from the electronic health record. Data pertaining to demographic information such as date of birth, sex, and co-morbidities were obtained. Additionally, clinical information on presentation, including clinical symptoms and vital signs, was collected. D-dimer values for patients were obtained, if available. The Wells score of patients was calculated retrospectively using the available clinical data. The CT-PA scans were reviewed by board-certified 


\section{Cureus}

radiologists to evaluate for the presence of filling defect suggestive of acute PE.

The scans were performed using the dual-source 128-slice scanner with the administration of $25 \mathrm{ml}$ nonionic contrast material with timed intravenous pump infusion followed by a $25-\mathrm{ml}$ saline flush, after the test bolus. The injection rate was $4 \mathrm{ml} / \mathrm{s}$ and the scan was performed from the diaphragm to the lung apices.

\section{Statistical analysis}

The collected data was combined using Microsoft Excel (Microsoft, Redmond, WA, USA). Categorical data, presented as frequencies and percentages, were compared using the chi-square or Fischer exact tests. Continuous variables, presented as means and standard deviations, were compared using the independent ttest or analysis of variance test. The statement of significance was based on a significant level of alpha at 0.05. A multivariable regression analysis model was conducted to identify the predictors of PE among patients with COVID-19. All the analysis was conducted using IBM SPSS for Windows, version 25 (IBM Corp., Armonk, NY, USA).

\section{Results}

\section{Patient characteristics}

The study included 316 patients, including 158 patients with COVID-19, who underwent CT-PA for ruling out PE. These included 200 (63.3\%) male patients and 116 (36.7\%) female patients. Most patients (72.2\%) were aged below 50 years. Overall, 152 (48.1\%) patients had a history of hypertension. Furthermore, 81 (25.6\%) patients were smokers and 57 (18.0\%) patients were obese. Only 40 (12.7\%) patients had a previous history of pulmonary disease.

Regarding the clinical presentation of patients, dyspnea was the most frequent symptom seen in 225 (71.2\%) patients. Fever was seen in 108 (34.2\%) patients. Furthermore, 84 (26.5\%) and 70 (22.2\%) presented with chest pain and cough, respectively. Only 32 (10.1\%) patients had an altered level of consciousness. In total, 218 (68.9\%) patients had tachycardia and 179 (56.6\%) had an oxygen saturation below $95 \%$.

Over half (54.4\%) of patients had elevated D-dimer levels. Leucocyte count was elevated in 204 (64.6\%) patients. There were 184 (58.2\%) and 160 (50.6\%) patients having an elevated erythrocyte sedimentation rate and C-reactive protein level, respectively (Table 1). 


\section{Cureus}

\begin{tabular}{|c|c|c|c|c|}
\hline \multicolumn{3}{|l|}{ Variable } & $\mathbf{N}$ & $\%$ \\
\hline \multirow{11}{*}{ Sociodemographic factors } & \multirow{4}{*}{ Age (years) } & 18-34 & 94 & 29.7 \\
\hline & & $35-49$ & 134 & 42.4 \\
\hline & & $50-65$ & 59 & 18.7 \\
\hline & & $>65$ & 29 & 9.2 \\
\hline & \multirow{2}{*}{ Gender } & Male & 200 & 63.3 \\
\hline & & Female & 116 & 36.7 \\
\hline & \multirow{4}{*}{ Comorbidities } & Hypertension & 152 & 48.1 \\
\hline & & Diabetes mellitus & 104 & 32.9 \\
\hline & & Pulmonary disease & 40 & 12.7 \\
\hline & & Obesity & 57 & 18.0 \\
\hline & \multicolumn{2}{|l|}{ Smokers } & 81 & 25.6 \\
\hline \multirow{7}{*}{ Clinical Features } & \multirow{5}{*}{ Presenting symptoms } & Dyspnea & 225 & 71.2 \\
\hline & & Chest pain & 84 & 26.5 \\
\hline & & Cough & 70 & 22.2 \\
\hline & & Fever & 108 & 34.2 \\
\hline & & Altered consciousness & 32 & 10.1 \\
\hline & \multicolumn{2}{|l|}{ Tachycardia } & 218 & 68.9 \\
\hline & \multicolumn{2}{|c|}{ Decreased oxygen saturation } & 179 & 56.6 \\
\hline \multirow{4}{*}{ Laboratory findings } & \multicolumn{2}{|c|}{ Elevated leucocyte count } & 204 & 64.6 \\
\hline & \multicolumn{2}{|c|}{ Elevated erythrocyte sedimentation rate } & 184 & 58.2 \\
\hline & \multicolumn{2}{|c|}{ Elevated C-reactive protein } & 160 & 50.6 \\
\hline & \multicolumn{2}{|l|}{ Elevated D-dimer } & 172 & 54.4 \\
\hline
\end{tabular}

\section{TABLE 1: Characteristics of patients}

$\mathrm{N}$, number of patients

\section{Comparison of the positive CT-PA rates}

Seventy-six patients were found to have PE on the CT-PA scan, yielding a positive rate of $24.1 \%$. However, there was a notable significant difference in the CT-PA positive rate according to the COVID-19 status. For example, only 13 (8.2\%) patients with COVID-19 were found to have PE while 63 (39.9\%) patients without COVID-19 had PE $(\mathrm{P}<0.01)$.

There was no statistically significant between patients with COVID-19 and those without COVID-19 regarding their age and gender $(\mathrm{P}>0.05)$. Furthermore, obesity was more prevalent among patients with COVID-19 (24.1\% vs. $12.0 \%$; P $<0.01)$. Similarly, diabetes mellitus was slightly more prevalent among patients with COVID-19 (36.1\% vs. 29.7\%), but the difference was not statistically significant $(\mathrm{P}=0.23)$.

Regarding the clinical and laboratory findings, fever was more commonly seen in patients with COVID-19 (51.9\% vs. $16.5 \%$; $\mathrm{P}<0.01)$. However, there was no significant difference in the prevalence of other presenting symptoms according to the COVID-19 status. Tachycardia was more frequent among patients without COVID-19 (87.3\% vs. 50.6\%; P < 0.01). In total, 138 (87.3\%) patients with COVID-19 had elevated Ddimer levels compared with 34 (21.5\%) patients without COVID-19 ( $\mathrm{P}<0.01)$. The difference between patients with COVID-19 and those without COVID-19 regarding the demographic, clinical, and laboratory findings is summarized in Table 2. 


\section{Cureus}

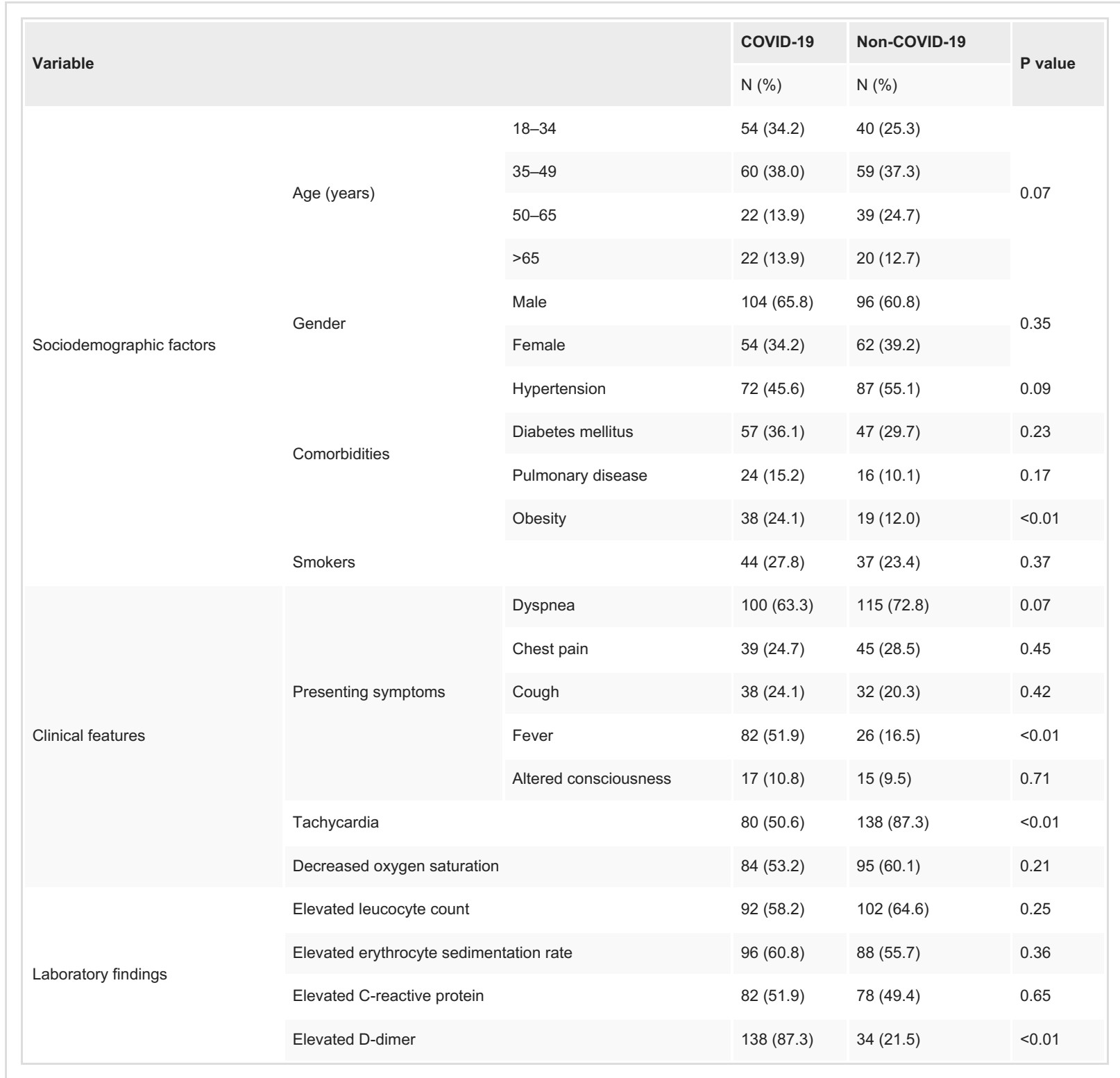

\section{TABLE 2: Characteristics of patients according to the COVID-19 status}

$\mathrm{N}$, number of patients; COVID-19, coronavirus disease 2019

\section{Multivariable analysis of factors associated with PE among COVID-19 patients}

A multivariable binary logistic regression analysis model was conducted to identify the independent factors associated with PE among patients with COVID-19. The model revealed that the smoking status (odds ratio $[\mathrm{OR}]=1.94 ; 95 \%$ confidence interval $[\mathrm{CI}]: 1.4-3.8$ ) and obesity (OR $=4.1 ; 95 \% \mathrm{CI}: 1.5-8.9)$ were independent predictors of PE. However, the elevated D-dimer level was not significantly associated with PE among patients with COVID-19 (OR $=0.7$; 95\% CI: 0.4-1.8).

\section{Discussion}

The study aimed to investigate the positive rate of CT-PA for PE in patients with COVID-19 and to identify the factors that serve as independent predictors of PE in such patients. The findings showed a significantly lower positive rate of CT-PA for PE among patients with COVID-19. Since coagulopathy became a wellrecognized complication of COVID-19, the lower positive rate of CT-PA in COVID-19 patients in our study may suggest the overutilization of CT-PA.

It is interesting to note that despite the lower positive rate for PE among patients with COVID-19, the elevated D-dimer levels were more common among patients with COVID-19. In keeping with the shared 
clinical features between severe COVID-19 pneumonia and PE, the elevated D-dimer level is likely a contributor to the overutilization of CT-PA for ruling out PE in such patients. Studies found that COVID-19 causes inflammation with endothelial injury resulting in elevated D-dimer levels [12]. Hence, D-dimer testing is insufficient to be a standalone test for the diagnosis of PE. Accordingly, some researchers suggested the use of different cut-off values for D-dimer in patients with COVID-19 [13].

The low positive rate of CT-PA for PE in the present study is consistent with previous studies. For example, Birk et al. conducted a retrospective study including 285 consecutive patients with suspected COVID- 19 who underwent CT-PA on a triage pathway [14]. Interestingly, they found that only one patient out of 48 patients with COVID-19 had PE compared with 25 patients out of 215 patients in the control group, suggesting that the prevalence of PE was lower in patients with COVID-19.

The study found that the smoking history is an independent factor associated with PE in patients with COVID-19. This is in line with previous literature which showed that current and former smokers are at higher risk of developing venous thromboembolism compared with non-smokers [15]. Therefore, interventional strategies aiming at increasing the awareness of the complications of COVID-19 among smokers are warranted.

To the best of our knowledge, this is the first study in Saudi Arabia to investigate the utilization of CT-PA in patients with COVID-19. However, the study has certain limitations. It is a retrospective study that may be limited by inaccurate or missing data about the clinical manifestations found in the electronic health records. Additionally, it is a two-center study and the results of this study may not be generalizable in all patients of COVID-19 due to variations in the practice and protocol of requesting medical imaging. Further studies are needed to confirm our findings.

\section{Conclusions}

This study suggests the probable overutilization of the CT-PA scans in patients with COVID-19 as indicated by the significantly lower CT-PA positive rate for PE among these patients. The significantly higher proportion of elevated D-dimer values above the normal reference range in these patients is a possible contributor. Hence, this finding should prompt physicians to consider a higher cut-off value for D-dimer in patients with COVID-19. Furthermore, it is essential to keep in mind that patients with COVID-19 who are smokers were found to be at higher risk of PE. The present study provides valuable information on the potential hazards of smoking among COVID-19 patients, and such information can be used to raise community awareness.

\section{Additional Information \\ Disclosures}

Human subjects: Consent was obtained or waived by all participants in this study. Imam Abdulrahman Bin Faisal University Institutional Review Board issued approval IAU-IRB 2020-155. Given the retrospective nature of the study, the need for informed consent was waived. Animal subjects: All authors have confirmed that this study did not involve animal subjects or tissue. Conflicts of interest: In compliance with the ICMJE uniform disclosure form, all authors declare the following: Payment/services info: All authors have declared that no financial support was received from any organization for the submitted work. Financial relationships: All authors have declared that they have no financial relationships at present or within the previous three years with any organizations that might have an interest in the submitted work. Other relationships: All authors have declared that there are no other relationships or activities that could appear to have influenced the submitted work.

\section{References}

1. Guan WJ, Ni ZY, Hu Y, et al.: Clinical characteristics of coronavirus disease 2019 in China . N Engl J Med. 2020, 382:1708-1720. 10.1056/NEJMoa2002032

2. Zheng KI, Feng G, Liu WY, Targher G, Byrne CD, Zheng MH: Extrapulmonary complications of COVID-19: a multisystem disease?. J Med Virol. 2021, 93:323-335. 10.1002/jmv.26294

3. Abobaker A, Raba AA, Alzwi A: Extrapulmonary and atypical clinical presentations of COVID-19. J Med Virol. 2020, 92:2458-2464. 10.1002/jmv.26157

4. Plasencia-Martínez JM, Rovira À, Caro Domínguez P, Barber I, García-Garrigós E, Arenas-Jiménez JJ: Extrathoracic manifestations of COVID-19 in adults and presentation of the disease in children . Radiologia (Engl Ed). 2021, 63:370-383. 10.1016/j.rxeng.2021.03.004

5. Perisetti A, Gajendran M, Mann R, Elhanafi S, Goyal H: COVID-19 extrapulmonary illness - special gastrointestinal and hepatic considerations. Dis Mon. 2020, 66:101064. 10.1016/j.disamonth.2020.101064

6. Liao D, Zhou F, Luo L, et al.: Haematological characteristics and risk factors in the classification and prognosis evaluation of COVID-19: a retrospective cohort study. Lancet Haematol. 2020, 7:E671-E678. 10.1016/S2352-3026(20)30217-9

7. Iba T, Levy JH, Levi M, Thachil J: Coagulopathy in COVID-19. J Thromb Haemost. 2020, 18:2103-2109. 10.1111/jth. 14975

8. Zhang X, Yang X, Jiao H, Liu X: Coagulopathy in patients with COVID-19: a systematic review and metaanalysis. Aging (Albany NY). 2020, 12:24535-24551. 10.18632/aging.104138 


\section{Cureus}

9. Middeldorp S, Coppens M, van Haaps TF, et al.: Incidence of venous thromboembolism in hospitalized patients with COVID-19. J Thromb Haemost. 2020, 18:1995-2002. 10.1111/jth.14888

10. Lodigiani C, Iapichino G, Carenzo L, et al.: Venous and arterial thromboembolic complications in COVID-19 patients admitted to an academic hospital in Milan, Italy. Thromb Res. 2020, 191:9-14.

10.1016/j.thromres.2020.04.024

11. Goldhaber SZ, Visani L, De Rosa M: Acute pulmonary embolism: clinical outcomes in the International Cooperative Pulmonary Embolism Registry (ICOPER). Lancet. 1999, 353:1386-1389. 10.1016/s01406736(98)07534-5

12. Mouhat B, Besutti M, Bouiller K, et al.: Elevated D-dimers and lack of anticoagulation predict PE in severe COVID-19 patients. Eur Respir J. 2020, 56:2001811. 10.1183/13993003.01811-2020

13. Korevaar DA, van Es J: Pulmonary embolism in COVID-19: D-dimer threshold selection should not be based on maximising Youden's index. Eur Respir J. 2021, 57:2004279. 10.1183/13993003.04279-2020

14. Birk R, Shaw D, Kennedy C, Higashi Y, Patel R, Gupta A, Au-Yong I: Low detection rate of pulmonary embolism in patients presenting to the emergency department with suspected coronavirus disease 2019 (COVID-19): a single-centre UK study. Curr Probl Diagn Radiol. 2021, 50:656-661. 10.1067/j.cpradiol.2020.09.014

15. Severinsen MT, Kristensen SR, Johnsen SP, Dethlefsen C, Tjønneland A, Overvad K: Smoking and venous thromboembolism: a Danish follow-up study. J Thromb Haemost. 2009, 7:1297-1303. 10.1111/j.15387836.2009.03490.x 